\author{
INOVAÇÃO NA EDUCAÇÃO \\ um olhar para os estilos de aprendizagem
}

\author{
Cleunisse Aparecida Rauen De Luca Canto ${ }^{1}$ \\ Universidade Federal de Santa Catarina \\ cleocanto@gmail.com \\ Rogério Cid Bastos ${ }^{2}$ \\ Universidade Federal de Santa Catarina \\ rogerio@inf.ufsc.br \\ Denise Maria Rengel ${ }^{3}$ \\ Faculdade de Tecnologia SENAI Joinville \\ denisemrv@sc.senai.br
}

\begin{abstract}
Resumo
Este estudo compara os resultados obtidos na aplicação de questionário sobre estilos de aprendizagem com achados da literatura, e correlaciona com as estratégias de ensino. Utilizamos os estudos obtidos na revisão realizada por Canto e Bastos (2019); aplicamos um questionário VAK (visual, auditory, kinesthetic) em 110 docentes-estudantes de uma pós-graduação e, na sequência, em 756 estudantes de diferentes modalidades; e comparamos os resultados obtidos. Dos 10 artigos selecionados, utilizaram instrumento de avaliação e focaram no padrão VAK, ou no VARK (incluindo aural-Read), no qual em $70 \%$ deles a preferência foi pelos estilos visual e cinestésico. Nesse estudo, $95 \%$ dos docentes-estudantes avaliados, e $80 \%$ nos demais estudantes, também demonstraram preferência pelos estilos visual e cinestésico. Concluímos que os estilos de aprendizagem permitem aos alunos identificar pontos fortes e fraquezas ao aprender, e aos professores definir as suas metas e estratégias de ensino para que consigam projetar atividades centradas no aluno.
\end{abstract}

Palavras-chave: Estilos de aprendizagem. Avaliação. Ambiente educacional. Inovação. Conhecimento.

\title{
INNOVATION IN EDUCATION
}

a look at learning styles

\begin{abstract}
The present study compares the results obtained from the application of a questionnaire on learning styles with the findings in the literature, in addition to identifying the existing correlations with teaching strategies for the management of educational knowledge. For that, we used the studies on learning styles obtained from a systematic literature review carried out by Canto and Bastos (2019); we applied a VAK questionnaire (visual, auditory, kinesthetic) to 110 teachers who were students of a postgraduate course and, subsequently, to 756 students distributed among technical, industrial learning, high school, and professional qualification courses; and compared the results obtained. The results showed that out of the 10 articles selected because they used an evaluation instrument and were focused on the VAK standard, or on the VARK (including Aural-Read), in $70 \%$ of them the preference was for the Visual and Kinesthetic styles. In this study, 95\% of the evaluated student-teachers and $80 \%$ of the other students also showed preference for the Visual and Kinesthetic styles. We concluded that exploring learning styles can enable students to identify strengths and weaknesses when learning and enable teachers to define their goals and activity strategies so that they can design student-centered activities.
\end{abstract}

Keywords: Learning styles. Evaluation. Educational environment. Innovation. Knowledge.

\footnotetext{
${ }^{1}$ Mestre em Psicopedagogia pela Universidade do Sul de Santa Catarina, Brasil (2004). Especialista de Ensino do SENAI - Departamento Regional de Santa Catarina.

${ }^{2}$ Doutor em Engenharia de Produção pela Universidade Federal de Santa Catarina, Especialista em Engenharia de Sistemas pela Universidade Técnica de Lisboa.

${ }^{3}$ Mestre em Educação pela Universidade da Região de Joinville - UNIVILLE, Especialista em Psicopedagia pela Faculdade Padre João Bagozzi.
} 


\section{INTRODUÇÃO}

A inovação é fundamental para o desenvolvimento sustentável, tanto em produtos quanto em processos, bem como na formação profissional. Apesar de o contexto educacional estar repleto de ações, propostas e práticas ditas 'inovadoras', grande parte delas já existem de forma sistemática desde o fim do século XIX e as primeiras décadas do século XX. Parece-nos plausível considerar esta inovação como uma renovação de práticas pedagógicas locais a partir de experiências inovadoras anteriores. A inovação, de modo geral, possui alguns pressupostos, a saber: é um processo incomum, não se faz a todo instante; precisa ser algo que proporcione reais condições às instituições e aos sistemas educativos; também precisa englobar um grande número de personagens que a atuam no cenário educacional e ser um processo planejado (CAMPOS, 2019).

Uma premissa importante na relação ensino e aprendizagem de conhecimentos, ou conteúdos, é que o que se ensina sempre está associado a metodologias, práticas, estratégias de ensino, atividades e recursos didáticos disponibilizados, sendo que o desafio está em transformar estes conhecimentos em algo com significado, relevantes, contextualizados, que envolvam os estudantes pela emoção e que permitam uma relação harmoniosa entre os saberes e as disciplinas, de forma interdisciplinar.

As complexas relações existentes no processo de ensino e de aprendizagem têm sido uma das temáticas que vem ganhando espaço no cenário educacional frente as diversas variáveis que envolvem essa questão, tal como ambiente educacional, comportamento dos acadêmicos e estilos de aprendizagem (SANTOS; BACINELLO; HEIN, 2017).

Em se tratando de estilos de aprendizagem, Dunn e Dunn (1978) ressaltaram que estes são um conjunto de condições por meio das quais os sujeitos concentram, absorvem, processam e retém informações e habilidades novas ou que consideram mais difíceis. São rotas que os indivíduos utilizam para o recebimento e o processamento das informações (KOLB, 1984).

Conforme apontado por Canto e Bastos (2019), a literatura apresenta distintas definições para o tema e diferentes instrumentos para identificação dos estilos de aprendizagem, cada um deles com uma classificação específica e que objetiva mensurar os tipos de capacidades mediadoras, de percepção de ordem, de julgamento, de entendimento, de processamento, entre outras inerentes ao processo de aprendizagem. Dentre os instrumentos comumente utilizados podemos destacar: 
- Inventário Myers-Briggs Type Indicator (MBTI), de Myers e Briggs (1970), que classifica os indivíduos em quatro dimensões (extrovertido/introvertido, sensorial/intuitivo, reflexivo/sentimental e julgador/perceptivo);

- Inventário dos Estilos de Aprendizagem de Kolb (1973), que considera quatro fases: experiência concreta (envolvimento), observação reflexiva (pensar sobre, ouvir e observar), conceituação abstrata (generalizações, ideias e teorias), e a experimentação ativa (testar e tomar decisões);

- Productivity Environmental Preference Survey (PEPS) de Dunn e Dunn (1978), que indica como os sujeitos respondem a estímulos ambientais, emocionais, sociais, físicos e psicológicos, categorias sob as quais estão agrupadas diferentes condições que afetam a aprendizagem;

- Style Delineator de Gregorc (1982), que mensura os tipos de capacidades mediadoras de percepção e de ordem em quatro estilos: sequencial concreto, sequencial abstrato, aleatório abstrato e aleatório concreto;

- Índice de Estilos de Aprendizagem de Felder e Silverman (1988), que abrange quatro dimensões da aprendizagem, considerando o nível de percepção (sensorial/intuitiva), o input (visual/verbal), o processamento (ativo/reflexivo) e o entendimento (sequencial/global);

- Modelo VARK (Visual, Aural-Read, Write and Kinesthetic) proposto por Fleming (2001), com uma técnica de mapeamento de estilos de aprendizagem composta pelos quatro canais de aprendizagem;

- Instrumento padronizado de Oxford (2003) que contém cinco seções, nomeadamente: 1) dedicada aos sentidos visuais, auditivos e cinestésicos da percepção; 2) estudar sozinho/introvertido, com outros/extrovertido); 3 ) intuição na tomada de decisão ou uso de pensamento analítico e de organização sequencial; 4) mentalidade fechada ou aberta e orientada para a descoberta; e 5) estilo de aprendizagem global (obtêm ideias principais) ou analítico (concentra-se mais em detalhes); e

- Questionário VAK (Visual, Auditory and Kinesthetic), um modelo elaborado na década de 1920 com o intuito de ajudar crianças com dislexia, desenvolvido por psicólogos como Fernald, Keller, Orton, Gillingham, Stillman e Montessor e que passou a ter relevância recentemente porque seus princípios e benefícios estendem-se a todos os tipos de aprendizagem e desenvolvimento. Segundo Ortiz (2011), está relacionado com a programação neurolinguística porque descreve como trabalha e se estrutura a mente 
humana, o que considera a via de ingresso da informação no cérebro (olho, ouvido e corpo).

Cada um dos questionários permite explorar os estilos de aprendizagem para identificar pontos fortes e fraquezas ao aprender, possibilitando aos professores observar, cuidadosamente, as estratégias de ensino para que consigam projetar atividades centradas no aluno.

É neste cenário que o presente estudo se pautou, tendo como objetivo comparar os resultados obtidos na aplicação de questionário sobre os estilos de aprendizagem com os achados da literatura, além de identificar as correlações existentes com as estratégias de ensino para a gestão do conhecimento educacional. A questão que norteou a pesquisa foi: "Que achados a literatura nos apresenta sobre os estilos de aprendizagem e qual a correlação com os dados obtidos em nosso estudo?" Para responder, utilizamos os dados obtidos na revisão sistemática da literatura realizada por Canto e Bastos (2019) e correlacionamos com o diagnóstico obtido em nosso estudo de caso para apontar as indicações quanto as estratégias de ensino.

Para tanto, o presente artigo foi estruturado para apresentar a revisão da literatura sobre o tema proposto, trazer de forma clara e objetiva o método utilizado para as buscas, apontar os resultados obtidos, correlacionar estes resultados com os achados da literatura e, finalmente, discorrer sobre as conclusões obtidas no estudo.

\section{REVISÃO DA LITERATURA}

Com as constantes mudanças que o mundo globalizando vem passando, a necessidade de inovar e de tornar mais eficiente o processo educacional faz com que o conhecimento sobre os estilos de aprendizagem seja fator primordial para auxiliar no aprimoramento do ensino.

O estilo de aprendizagem (EA), segundo Felder e Silverman (1988), pode ser definido como o modo pelo qual os indivíduos preferem receber e processar as informações. Estão relacionados com a forma particular de adquirir conhecimentos, habilidades e atitudes através da experiência, ou de anos de estudos, e levam em consideração a hereditariedade (código genético), a educação, a personalidade e a adaptação do indivíduo às demandas do ambiente (SILVA; WESCHELR, 2010).

A preferência pelo estilo de aprendizagem deu margem, ao longo dos anos, para a criação de distintos modelos que pudessem medir as dimensões desses estilos. Cada um deles, porém, captando e analisando um conjunto de indicadores em uma diversidade de 
possibilidades, alguns com nomenclaturas diferentes, mas sendo similares nas análises, na maioria das vezes.

Um dos modelos de estilo de aprendizagem mais popular é o modelo que foi desenvolvido por Neil Fleming em 1987. Esse modelo identifica quatro tipos primários de estilos de aprendizado: visual (visual), auditivo (auditory), leitura/gravação (read) e cinestésico (kinesthetic), onde as iniciais de cada preferência dão nome ao Modelo VARK (FLEMING; BAUME, 2006). O modelo sugere que cada aprendiz tem diferentes preferências de aprendizagem para as quais responde melhor do que outras.

Similar a este modelo, mas com foco em três estilos (Visual, Auditory, Kenesthetic), a programação neurolinguística aponta que estes ajudam na recepção e assimilação da informação, sendo comum que um destes estilos seja o preferido na obtenção da informação, ou ainda que estes possam estar uniformemente equilibrados e possuir os três, não apresentando uma maior predominância ou preferência (MEHAY, 2010).

A revisão sistemática da literatura desenvolvida por Canto e Bastos (2019), aprovada para o ICKM|SUCEG 2019 (15th International Conference on Knowledge Management \& II Seminar of Corporate Universities and Government, 2019), apresentou 10 artigos, dos 16 totais, que focaram nas preferências de aprendizagem baseado no Modelo VARK, ou VAK (quando o padrão de leitura/gravação não é avaliado). Estes artigos apresentaram a aplicação de diferentes instrumentos de avaliação para medir os estilos de aprendizagem, delimitaram o público alvo e detalharam os resultados obtidos, conforme podemos visualizar no Quadro 1.

Quadro 1 - Relação dos estudos obtidos de revisão sistemática da literatura

\begin{tabular}{|c|c|c|c|}
\hline Autor & Amostra & $\begin{array}{c}\text { Instrumento de } \\
\text { Avaliação }\end{array}$ & Resultados \\
\hline $\begin{array}{l}\text { ALKOOHEJI; AL- } \\
\text { HATTAMI, } 2018\end{array}$ & $\begin{array}{l}185 \text { estudantes da } \\
\text { University of } \\
\text { Bahrain, em } \\
\text { Bahrain }\end{array}$ & $\begin{array}{l}\text { Questionário } \\
\text { VARK online }\end{array}$ & $\begin{array}{c}\text { Geral: Cinestésico (38\%), Visual (37\%), } \\
\text { Auditivo (32\%) e Leitura/Escrita (27\%). } \\
\text { Masculino: visual (29\%) e cinestésica } \\
\text { (27\%). Feminino: cinestésica }(28 \%) \text { e } \\
\text { visual. (27\%) A diferença entre as } \\
\text { preferências masculina e feminina não é } \\
\text { significante. }\end{array}$ \\
\hline $\begin{array}{l}\text { ALRABAH; WU; } \\
\text { ALOTAIBI, } 2018\end{array}$ & $\begin{array}{l}250 \text { acadêmicos de } \\
\text { 'inglês como } \\
\text { língua estrangeira' } \\
\text { no Kuwait }\end{array}$ & $\begin{array}{l}\text { Instrumento } \\
\text { padronizado por } \\
\text { Oxford (2003), } \\
\text { online }\end{array}$ & $\begin{array}{l}\text { Os estilos dominantes nas cinco categorias } \\
\text { foram o visual }(36,75 \%) \text { e o cinestésico } \\
(34,29 \%) \text {; o extrovertido (60,65\%); o } \\
\text { intuitivo }(50,68 \%) \text {; o concreto-sequencial } \\
(58 \%) \text {; e o de fechamento-orientado e } \\
\text { global }(56,80 \%) \text {. }\end{array}$ \\
\hline AYCAN, 2018 & $\begin{array}{l}21 \text { acadêmicos em } \\
\text { formação } \\
\text { pedagógica de } \\
\text { uma Universidade }\end{array}$ & $\begin{array}{l}\text { O teste online com } \\
\text { três categorias e } 20 \\
\text { questões VAK }\end{array}$ & $\begin{array}{l}17(51,5 \%) \text { aprende vendo (visual), } \\
9(27,5 \%) \text { aprende fazendo (cinestésico) } \\
7(21,2 \%) \text { aprende ouvindo (auditivo) }\end{array}$ \\
\hline $\begin{array}{c}\text { BOSMAN; } \\
\text { SCHULZE, } 2018\end{array}$ & $\begin{array}{l}240 \text { aprendizes de } \\
\text { matemática em }\end{array}$ & $\begin{array}{l}\text { Questionário } \\
\text { VARK, } \\
\text { Aprendizagem } \\
\end{array}$ & $\begin{array}{c}\text { A preferência pela aprendizagem } \\
\text { individual diferenciou significativamente } \\
\text { os aprendizes. }\end{array}$ \\
\hline
\end{tabular}




\begin{tabular}{|c|c|c|c|}
\hline & $\begin{array}{l}\text { uma Universidade } \\
\text { da África do Sul }\end{array}$ & $\begin{array}{l}\text { individual e grupal } \\
\text { e entrevistas } \\
\text { gravadas } \\
\end{array}$ & $\begin{array}{c}\text { A correlação entre a aprendizagem visual e } \\
\text { auditiva foi significativa. }\end{array}$ \\
\hline $\begin{array}{l}\text { DINCOL-OZGUR, } \\
2018\end{array}$ & $\begin{array}{l}251 \text { futuros } \\
\text { professores de } \\
\text { química e ciências, } \\
\text { entre } 18 \text { e } 23 \text { anos } \\
\text { de idade, de três } \\
\text { Universidades }\end{array}$ & $\begin{array}{l}\text { Inventário de estilo } \\
\text { de aprendizagem } \\
\text { Maggie McVay } \\
\text { Lynch (padrão } \\
\text { VAK) }\end{array}$ & $\begin{array}{l}61,8 \% \text { dos futuros professores tinham um } \\
\text { estilo de aprendizagem visual, seguido por } \\
\text { cinestésico }(19,9 \%) \text { e auditivo }(18,3 \%)\end{array}$ \\
\hline $\begin{array}{l}\text { GULNAZ; } \\
\text { FAROOQ; ALI, } \\
2018\end{array}$ & $\begin{array}{l}200 \text { estudantes de } \\
\text { English as a } \\
\text { Foreign Language } \\
\text { (EFL) da Arábia } \\
\text { Saudita na Taif } \\
\text { University }\end{array}$ & $\begin{array}{l}\text { Pesquisa de opinião } \\
\text { (uso dos sentidos, } \\
\text { intelecto, força e } \\
\text { movimentos físicos. }\end{array}$ & $\begin{array}{c}\text { 'Eu gosto de instruções escritas para uma } \\
\text { tarefa'. Preferência pelo aprender por meio } \\
\text { de imagens, gráficos, diagramas, mapas e } \\
\text { vídeos ao invés de outros meios de } \\
\text { comunicação ou instruções orais, sendo } \\
\text { que ler e escrever foi o segundo estilo mais } \\
\text { preferido. }\end{array}$ \\
\hline $\begin{array}{l}\text { HUANG; HOI; } \\
\text { TEO, } 2018\end{array}$ & $\begin{array}{l}329 \text { estudantes de } \\
\text { inglês chineses de } \\
\text { uma universidade } \\
\text { no leste da China }\end{array}$ & $\begin{array}{c}\text { Estilo de } \\
\text { aprendizagem de } \\
\text { Cohen et al. (1995) }\end{array}$ & $\begin{array}{c}\text { Os estudantes universitários chineses } \\
\text { preferiram o estilo de aprendizagem visual, } \\
\text { seguido pelos estilos auditivos e } \\
\text { cinestésico. }\end{array}$ \\
\hline $\begin{array}{l}\text { HUSMANN; } \\
\text { O'LOUGHLIN, } \\
2019\end{array}$ & $\begin{array}{l}426 \text { acadêmicos de } \\
\text { anatomia da } \\
\text { graduação }\end{array}$ & $\begin{array}{l}\text { Questionário VARK } \\
\text { + Pesquisa sobre a } \\
\text { estratégia de estudo } \\
\text { utilizada }\end{array}$ & $\begin{array}{l}\text { A maioria dos alunos não relatava } \\
\text { estratégias de estudo que se } \\
\text { correlacionavam com seus VARK de } \\
\text { avaliação, o desempenho em anatomia não } \\
\text { foi correlacionado com a sua pontuação } \\
\text { em qualquer categoria de VARK e não } \\
\text { teve nenhuma correlação com os } \\
\text { resultados do curso da anatomia. }\end{array}$ \\
\hline $\begin{array}{l}\text { SENER; } \\
\text { ÇOKÇALISKAN, } \\
2018\end{array}$ & $\begin{array}{l}88 \text { alunos do } \\
\text { ensino médio do } \\
\text { curso de inglês } \\
\text { como língua } \\
\text { estrangeira (EFL) }\end{array}$ & $\begin{array}{ll}\text { Questionário } & \text { de } \\
\text { preferências } & \text { de } \\
\text { estilo } & \text { de } \\
\text { aprendizagem } & \\
\text { perceptual (PLSPQ) } \\
\text { e o Inventário de } \\
\text { inteligência }\end{array}$ & $\begin{array}{l}\text { Os estilos tátil e auditivo foram os mais } \\
\text { frequentes entre os estudantes, sem } \\
\text { diferenças significativas quanto ao gênero. } \\
\text { Visual e cinestésico se destacaram nas } \\
\text { inteligências múltiplas. }\end{array}$ \\
\hline ÜNSAL, 2018 & $\begin{array}{l}48 \text { estudantes que } \\
\text { estavam } \\
\text { aprendendo } \\
\text { francês na escola } \\
\text { de línguas } \\
\text { estrangeiras na } \\
\text { Universidade de } \\
\text { Mármara }\end{array}$ & $\begin{array}{l}\text { Questionário do } \\
\text { modelo de estilos de } \\
\text { aprendizagem de } \\
\text { 'Dunn \& Dunn' }\end{array}$ & $\begin{array}{l}50 \% \text { eram aprendizes visuais, } 12,5 \% \\
\text { auditivos, e } 22,91 \% \text { cinestésicos. } \\
\text { Além disso, para } 14,58 \% \text { dos estudantes, } \\
\text { dois ou três estilos de aprendizagem } \\
\text { coexistem, sendo } 4,16 \% \text { visuais-auditivos, } \\
6,25 \% \text { visual-cinestésicos e } 4,16 \% \text { visual- } \\
\text { auditivo-cinestésicos. }\end{array}$ \\
\hline
\end{tabular}

Fonte: Adaptado de Canto \& Bastos (2019).

Na percepção de Canto e Bastos (2019), as preferências pelos estilos de aprendizagem dos estudantes devem se refletir nas estratégias de ensino dos professores, já que essas são direcionadoras do processo de aprendizagem dos estudantes na visão da maior parte dos autores.

Analisando a revisão sistemática da literatura de Mendes e Bottentuit Júnior (2015), onde o foco foi publicações stricto sensu disponíveis nos Bancos de Teses e Dissertações da CAPES e do IBCTI durante o período de 2010 a 2014, e também relacionadas aos estilos de aprendizagem, os autores apontaram que: 


\section{ARTIGO}

INOVAÇÃo

- a partir de 2004 os estudos sobre os EA aumentaram, sendo possível observar um tímido interesse pela área em anos anteriores;

- 28 produções acadêmicas focaram em EA, de acordo com os critérios pré-estabelecidos, e estavam disponíveis nos BDTD da CAPES e do IBCTI, sendo 20 dissertações e 8 teses;

- o ano de 2012 foi o ápice, com 16 produções;

- a predominância foi na educação, alcançando um índice de 24,6\% (14\% Educação, 7\% Educação e Matemática Tecnológica e 3,6\% Psicologia Escolar e do Desenvolvimento Humano), disparando em relação às outras áreas;

- $\quad 42,8 \%$ presentes no âmbito da educação superior, seja apenas uma análise direcionada ao reconhecimento das preferências desse público ou para verificar se há ocorrência de estratégias que inserem os EA na perspectiva de ensino;

- 67,9\% das amostras eram constituídas por pesquisas com estudantes universitários e $10,7 \%$ com docentes;

- 46,4\% apontou o uso de instrumento como Questionário/Inventário;

Assim, se correlacionarmos os dados apontados nos estudos de Canto e Bastos (2019) com os de Mendes e Bottentuit Júnior (2015) podemos observar que para ambos o maior foco esteve na educação, que os estudantes universitários computaram o maior índice das amostras, logo com ações direcionadas para o ensino superior, e com um número significativo de estudos utilizando algum instrumentos de avaliação para medir os estilos de aprendizagem.

Em suma, é "[...] imperativo ensinar os alunos a adotar e incorporar progressivamente estratégias de aprendizagem, ensinar-lhes a ser mais conscientes sobre a forma como aprendem, para que possam enfrentar satisfatoriamente diversas situações de aprendizagem" (VILLAVICENCIO, 2004, p. 4), ganhando assim autonomia para compreender e apreender de forma consciente e por meio de estratégias focadas nos seus estilos de aprendizagem.

\section{MÉTODO}

Para a correlação deste estudo, utilizamos a revisão sistemática da literatura de Canto e Bastos (2019). A revisão contou com buscas na base de dados ERIC (Education Resources Information Center), por se tratar de uma biblioteca digital de pesquisa e informação sobre educação. As buscas foram realizadas no mês de fevereiro de 2019 com foco em 'estilos de aprendizagem'. Para a seleção dos artigos os autores focaram em artigos 'open source', com indicação em EA, exposição baseada em análises qualitativas, quantitativas, ou ambas, com uso 
de instrumentos de avaliação e desfecho apontando aplicação na educação. Dos 36 artigos 'open source' selecionados, 20 estudos atendiam aos critérios de inclusão e 10 serviram para correlacionar os resultados com os dados obtidos em nosso estudo de caso.

\section{$\underline{\text { O Estudo de caso }}$}

Como parte das atividades de uma das disciplinas de um curso de Pós-Graduação Lato Sensu, na área da educação, em uma Instituição de Ensino Superior de Florianópolis, identificamos os estilos de aprendizagem dos alunos seguindo uma abordagem de pesquisa quantitativa, por meio de um Questionário VAK.

Esta é a primeira disciplina ministrada no curso e tem como pré-requisito abordar temas focados em como o cérebro aprende, em modelos de aquisição de conhecimento, na importância da experiência no contexto do aprendizado, nos processos de cognição e metacognição (neurociência), e na educação inclusiva. A disciplina possuía uma carga horária de 40h, sendo que 16h eram destinadas a imersão (presenciais) e 24 remota (EaD). Por ser um curso na área da educação, um dos requisitos para a seleção dos alunos foi que todos estivessem atuando como docentes em algum curso da instituição.

A disciplina foi ministrada para 121 alunos em três turmas distintas: a Turma I com 34 alunos (29\%); a Turma II com 44 (36\%); e a Turma III com 43 (35\%). Todos, durante a primeira imersão da disciplina responderam ao questionário VAK para identificação do seu estilo de aprendizagem. Para a 'Turma III', os alunos, também docentes em algum curso da Instituição, foram desafiados a aplicar o mesmo questionário para identificar o estilo de aprendizagem dos seus alunos.

O modelo VAK possui um questionário através do qual é possível avaliar os estilos de aprendizagem das pessoas de acordo com as suas preferências. Assim, pessoas com estilo visual são caracterizadas como as que preferem ver e ler, além de observar e analisar a partir de imagens, diagramas, gráficos, filmes, entre outros. Pessoas com estilo auditivo têm predileção pela escuta e pelo diálogo. E por fim, as pessoas cinestésicas demonstram preferência por tocar e fazer para assimilarem a informação, o que significa que a aprendizagem envolve a experiência física, o sentir o experimentar, o vivenciar, ou seja, literalmente 'colocar a mão na massa'.

Em nosso estudo utilizamos um questionário com 10 questões e três possíveis respostas $(a, b, c)$, sendo que aquela que mais se aproximasse do estudante valia 10, a que menos se relacionasse com o estudante valia 1 e aquela que correspondia a um item mais ou menos próximo valia 5. Ao final, cada estudante precisou computar qual a pontuação obtida, para tanto 
ele foi convidado a somar todos os pontos da resposta 'a', bem como todos para 'b' e depois para 'c'. O somatório das respostas obtidas indicava se o estudante era visual (pontuação de ' $a$ '), auditivo (pontuação de 'b') ou cinestésico (pontuação de 'c'). Sempre que a pontuação se igualava era considerado preferências múltiplas.

Após aplicação do questionário utilizamos o aporte de Nunan (1996) para explicar ao público alvo cada estilo de aprendizagem, com isto permitindo alertá-los que as experiências as quais estamos expostos podem ajudar a determinar maneiras privilegiadas de aprender.

\section{RESULTADOS}

Dos 121 alunos da pós-graduação lato sensu, 110 (90\%) conseguiram finalizar o questionário VAK. Destes, 62 (56\%) alunos apresentaram estilo de aprendizagem cinestésica, $43(39 \%)$ visual e $5(5 \%)$ auditivo. Os resultados mostraram baixíssima preferência pelo auditivo, conforme podemos visualizar no gráfico a seguir.

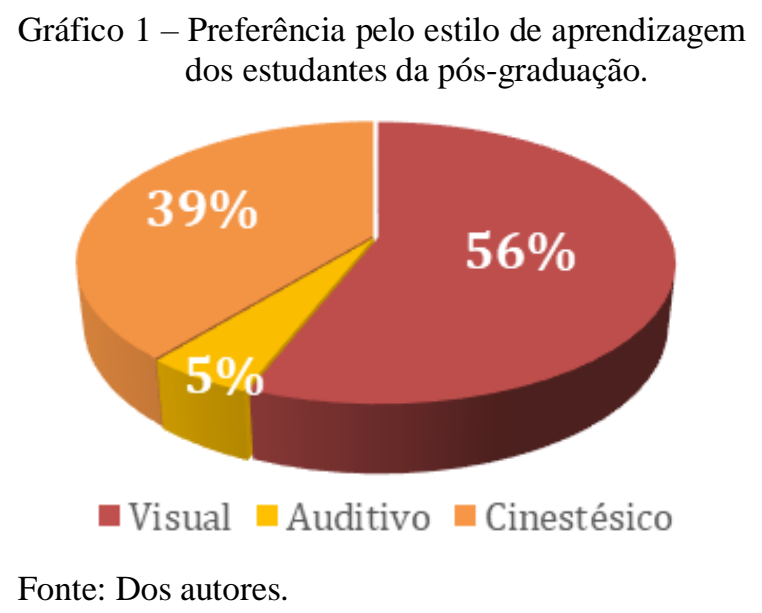

Após identificarmos os estilos de aprendizagem dos estudantes da pós-graduação lato sensu, desafiamos os 43 alunos da 'Turma III', que também eram professores, a aplicar o mesmo questionário em suas aulas, com seus alunos, como uma das tarefas de aprendizagem propostas durante a aprendizagem remota. Assim, dos 43 alunos da turma, 34 (79\%) conseguiram finalizar a atividade no prazo e aplicaram a atividade com 756 alunos, sendo 538 (72\%) dos cursos de aprendizagem industrial, 117 (15\%) dos cursos técnicos (estudantes que estão cursando ou concluíram o ensino médio) e 101 (13\%) dos cursos de qualificação ou de iniciação profissional, de diferentes idades e formação. Não houve aplicação com os estudantes do ensino superior porque nenhum dos docentes desta turma atuava nesta modalidade na Instituição. O Gráfico 2 apresenta uma síntese dos resultados obtidos. 
Gráfico 2 - Estilo de aprendizagem dos estudantes envolvidos na análise.
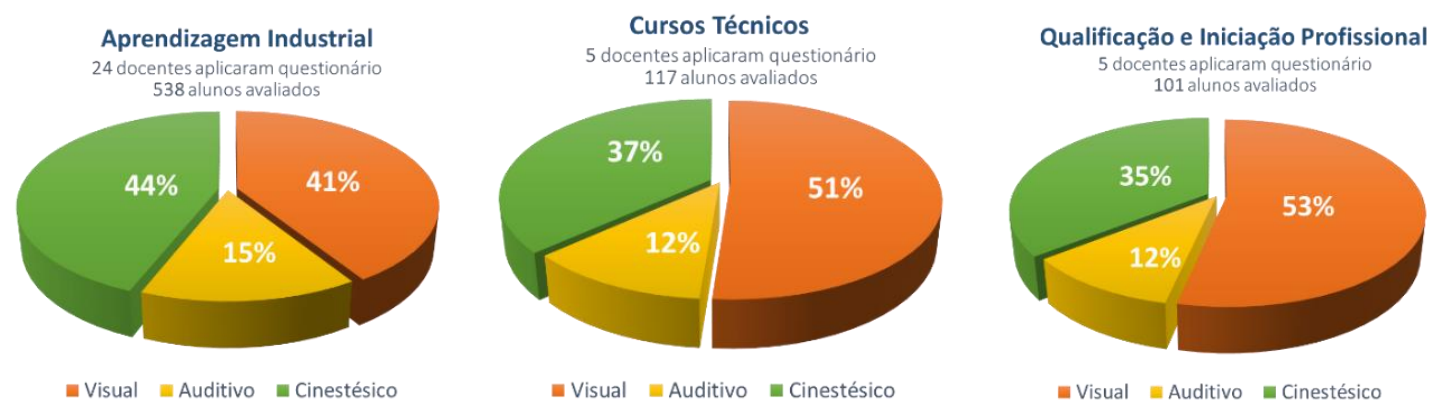

Fonte: Dos autores.

Conforme podemos observar, os gráficos apontam que, proporcionalmente para todos os cursos avaliados, o estilo de aprendizagem com menor representação é o auditivo, sendo mais de $80 \%$ da preferência, em cada modalidade, pelo visual e o cinestésico. Para o curso de aprendizagem industrial, em que o número maior de estudantes possui menor idade (entre $14 \mathrm{e}$ 24 anos), o cinestésico foi o estilo que sobresaiu; enquanto nos cursos técnicos, qualificação e iniciação profissional foi o visual.

Importante ressaltar que os cursos aos quais estes estudantes estão vinculados é de uma Instituição de Educação Profissional, com uma metodologia baseada em competências e com grande foco no 'saber fazer', o que pode justificar a grande preferência pelos estilos visual e cinestésico.

\section{DISCUSSÃO DOS RESULTADOS}

Os estudos sobre os estilos de aprendizagem apontaram para questões significativas sobre o processo de ensino e de aprendizagem, tanto em termos de preferências de aprendizagem quanto sobre os diferentes instrumentos de avaliação (questionários/inventários) e as percepções sobre o tema.

Quando correlacionamos os estudos obtidos por meio de uma revisão sistemática da literatura de Canto e Bastos (2019) com os achados deste estudo pudemos observar que os resultados obtidos não diferem no foco e nem nas preferências por aprendizagem.

Nosso estudo utilizou o questionário VAK como principal método de avaliação dos estilos de aprendizagem, focando nas preferências visual, auditivo e cinestésico, o que está de acordo com os estudos de Alkooheki e Al-Hattami (2018) e Gulnaz; Farooq; Ali (2018). Segundo Mendes e Bottentuit Júnior (2015), 46,4\% dos estudos obtidos na revisão sistemática 
da literatura adotaram um instrumento de avaliação para identificar preferências por aprendizagem.

Quando avaliamos só os alunos da pós-graduação lato sensu, todos adultos, identificamos que $56 \%$ deles têm preferência cinestésica e $39 \%$ visual, totalizando $95 \%$ com estas preferências combinadas. Estes mesmos achados podem ser visualizados nos estudos de Dincol-Ozgur (2018), onde 81,7\% da preferência dos professores de química e ciências foi pelos estilos visual e cinestésico. Ünsal (2018) também encontrou resultados similares, pois $73 \%$ dos aprendizes de francês da Universidade de Marmara demonstraram preferência pelos estilos visual e cinestésico; da mesma forma que Alrabah et al. (2018), que alcançou 71\% da preferência dos estudantes universitários e Aycan (2018), que obteve 78\% da preferência dos estudantes do programa de certificação em formação pedagógica de uma Universidade.

O cenário não alterou quando o foco mudou, ou seja, quando os estudantes da pósgraduação aplicaram o questionário sobre os estilos de aprendizagem para os estudantes dos cursos de aprendizagem industrial, curso técnico, curso de qualificação e de iniciação profissional, pois a preferência pelo visual e o cinestésico foi de, aproximadamente, $80 \%$.

Nos estudos de Ünsal (2018), os múltiplos estilos também foram apontados, já que $14,58 \%$ dos aprendizes demonstraram dois ou três estilos de aprendizagem coexistindo, distribuídos entre visual-auditivo $(4,16 \%)$, visual-cinestético $(6,25 \%)$ e visual-auditivocinestético $(4,16 \%)$.

Conforme reforçou Alkooheji e Al-Hattami (2018), o fato de o estilo de aprendizagem auditiva não prevalecer em seus estudos foi algo alarmante, já que o principal método de ensino da instituição era auditivo. Em nosso estudo, porém, o fato de o auditivo não prevalecer é aceitável, já que os estudantes participam de aulas focadas no desenvolvimento de competências e pautadas nos quatro pilares da educação no século XXI, o "aprender a conhecer", o "aprender a fazer", o "aprender a ser" e o "aprender a conviver" (DELORS, 2012) e com muita atividade prática.

Huang et al. (2018) destacaram a preferência visual, mas seguida do auditivo. Para os autores, a preferência pelo estilo de aprendizagem visual pode ser atribuída a razões culturais, já que a ênfase da educação tradicional chinesa é a memória de aprendizagem através da escrita. Sener e Çokçaliskan (2018) obtiveram preferência pelo estilo cinestésico (tátil), mas seguido do auditivo, e sem diferenças significativas quanto ao gênero.

A falta de correlação entre os estilos de aprendizagem e as estratégias de estudo foi citada por Günes (2018) e Husmann e O'Loughlin (2019). Para estes autores os professores não 
devem promover o conceito de estilos de aprendizagem para estudo ou para intervenções de ensino, pois acreditam que estes não interferem no processo.

Com relação as estratégias de ensino, a revisão sistemática da literatura de Canto e Bastos (2019) apontou que:

- as estratégias educacionais devem coincidir com os EA dos estudantes, objetivando que os alunos compreendam projetos de acordo com suas abordagens de aprendizado (AYCAN, 2018);

- os EA visual e cinestésico requerem professores criativos para beneficiar a aprendizagem, enquanto os auditivos professores pacientes, que explicam detalhadamente e que forneçam exemplos (BOSMAN; SCHULZE, 2018);

- os EA desempenham papéis significativos na formação de competências de aprendizagem autorregulada, por isso é importante considerar esta correlação quando se cria estratégias de aprendizado (DINCOL-OZGUR, 2018);

- as diferenças individuais e as de EA devem ser levadas em consideração pelos professores para criar estratégias de ensino (ELBAN, 2018);

- os professores devem fazer uso dos EA e projetar estratégias que promovam a aprendizagem (GULNAZ; FAROOQ; ALI, 2018);

- os cursos podem ser configurados, e os programas de treinamento organizados, para que o ensino esteja de acordo com EA dos estudantes (ÜNSAL, 2018).

Precisamos considerar que experimentar, refletir, pensar e agir, segundo mencionou Kolb e Kolb (2017), estão intimamente relacionados entre si, são modos independentes, mas variam para diferentes indivíduos e para suas formas de aprendizagem. O que se faz necessário, no entanto, é que os estilos de aprendizagem estejam no pensamento dos professores e dos estudantes, assim as estratégias de ensino podem se adaptar ao tipo de aprendizado preferido dos estudantes.

\section{CONSIDERAÇÕES FINAIS}

Avaliar os estilos de aprendizagem é de bastante relevância atualmente, já que permite direcionar professores e estudantes no ambiente educacional.

A correlação entre os achados deste estudo com uma revisão sistemática da literatura trouxe respostas para a questão de pesquisa e nortearam no sentido de adaptação das técnicas de ensino ao tipo de aprendizado preferido dos aprendizes. 


\section{ARTIGO}

INOVAÇÃO

A experiência de aprendizado precisa levar em consideração as orientações visuais, as tendências e as percepções sensoriais, o saber fazer, para que a aula seja motivadora. Isto ficou bem evidenciado em todos os estudos, já que o visual e o cinestésico se sobressaíram nas preferências de estilos de aprendizagem dos estudantes na maior parte deles.

A inovação é o principal motivador na conclusão dos diversos autores, já que os estudos apontaram a necessidade de os professores incorporarem no dia a dia de sala de aula atividades que envolvam os alunos (visuais), que trabalhem em grupos (interpessoais), e que permitam colocar a mão na massa, hands on (cinestésica).

Os resultados apontaram ser necessário que o aprendizado seja ativo, que os alunos tenham oportunidade de regular seus processos de aprendizagem, que passem de ouvintes passivos e anotadores a exploradores conscientes, que investiguem relacionamentos entre fatos e implementem comparações com informações conflitantes.

Concluímos que mesmo que os autores da revisão sistemática tenham utilizado diferentes instrumentos para avaliar, os achados deste estudo estão em consonância com os obtidos na literatura. Corroboramos com a literatura que o conhecimento sobre os estilos de aprendizagem dos estudantes é estratégia relevante para auxiliar no processo de ensino, pois permite os professores melhorar as suas metas e estratégias de atividades para que consigam projetar atividades centradas no aluno. 


\section{ARTIGO}

INOVAÇÃo

\section{REFERÊNCIAS}

ALKOOHEJI, L.; AL-HATTAMI, A. Learning Style Preferences among College Students. International Education Studies, v. 11, n. 10, p. 50-63, 2018.

ALRABAH, S.; WU, S.-H.; ALOTAIBI, A. M. The Learning Styles and Multiple Intelligences of EFL College Students in Kuwait. International Education Studies, v. 11, n. 3, p. 38-47, 2018.

AYCAN, K. Discovering Learning Style with Active Music Education Practices. Cypriot Journal of Educational Sciences, v. 13, n. 4, p. 562-576, 2018.

BOSMAN, A.; SCHULZE, S. Learning Style Preferences and Mathematics Achievement of Secondary School Learners. South African Journal of Education, v. 38, n. 1, 2018.

CANTO, C. A. R. DE L.; BASTOS, R. C. Avaliação dos estilos de aprendizagem em universitários: uma revisão sistemática, Knowledge and Learning Governance for Innovation, ICKM/SUCEG, Anais, 2019. [no prelo].

CAMPOS, F. R. Inovação ou renovação educacional? Dilemas, controvérsias e o futuro da escolarização. In: CAMPOS, F. R.; BLIKSTEIN, P. (orgs.). Inovações radicais na educação brasileira. Porto Alegre, RS: Penso, 2019.

DELORS, J. (org.). Educação um tesouro a descobrir - Relatório para a Unesco da Comissão Internacional sobre Educação para o Século XXI. Editora Cortez, 7ª edição, 2012.

DINCOL-OZGUR, S. Effect of Learning Styles on Prospective Teachers' Self-Regulated Learning Skills. Cypriot Journal of Educational Sciences, v. 13, n. 4, p. 521-528, 2018.

DUNN, R.; DUNN, K. Ensinando alunos através de seus estilos individuais de aprendizagem. Reston, VA: Reston, 1978.

ELBAN, M. Learning Styles as the Predictor of Academic Success of the Pre-Service History Teachers. European Journal of Educational Research, v. 7, n. 3, p. 659-665, 2018.

FELDER, R. M.; SILVERMAN, L. K. Learning and Teaching Styles in Engineering Education. Journal of Engineering Education, v. 78, n. 7, p. 674-681, 1988.

FLEMING, N.D.; MILLS, C. Not Another Inventory, Rather a Catalyst for Reflection. To Improve the Academy, v. 11, p. 137-155, 1992.

FLEMING, N. D. Teaching and learning styles: VARK strategies. Christchurch, New Zealand: N. D. Fleming, 2001.

FLEMING, N; BAUME, D. Learning styles again: VARKing up the right tree! Educational Developments, SEDA Ltd, p. 4-7, Nov. 2006.

GREGORC, A F. Estilos de aprendizagem/ensino: Potentes forças por trás deles. Liderança Educacional, v. 5, p. 234-237, 1979. 


\section{ARTIGO}

INOVAÇÃo

GULNAZ, F.; FAROOQ, M. U.; ALI, S. Learning Styles: Preferred Learning Choices and Behaviors of Saudi Male and Female EFL Learners. Journal of Education and Educational Development, v. 5, n. 2, p. 60-77, 2018.

GÜNES, M. H. Learning Styles of the Students of Biology Department and Prospective Biology Teachers in Turkey and Their Relationship with Some Demographic Variables. Universal Journal of Educational Research, v. 6, n. 3, p. 366-377, 2018.

HUANG, F.; HOI, C. K. W.; TEO, T. The Influence of Learning Style on English Learning Achievement among Undergraduates in Mainland China. Journal of Psycholinguistic Research, v. 47, n. 5, p. 1069-1084, 2018.

KOLB, D. On management and the learning process. Working paper Sloan School of Management, Cambridge, 70p., 1973.

KOLB, D. Experiential learning. Englewood Cliffs, New Jersey: Prentice Hall, 1984.

KOLB, A. Y.; KOLB, D. A. Experiential Learning Theory as a Guide for Experiential Educators in Higher Education. A Journal for Engaged Educators, v. 1, n. 1, p. 7-44, 2017.

MEHAY, R. Honey \& Mumford Learning Styles \& VAK model. Bradford. 2010. Disponível em: http://www.essentialgptrainingbook.com/resources/chapter_10. Acesso em dezembro de 2019.

MENDES, A. G. L. M.; BOTTENTUI JUNIOR, J. B. Revisão Sistemática da Literatura (RSL) das Teses e Dissertações produzidas acerca dos estilos de aprendizagem. Revista EducaOnline, v. 9, n. 2, mai/ago, 2015.

NUNAN, D. Taking control autonomy in language learning. Hong Kong: Hong Kong Universtiy Press. 1996. Disponível em: https://books.google.com.br/books?hl=ptBR\&lr=\&id=pTHxAQAAQBAJ\&oi=fnd\&pg=PA13\&dq=david+nunan+(1996). Acesso em dezembro 2019.

ORTIZ, J. V. El conocimiento de los estilos de aprendizaje como medida de atención a la diversidad y sus implicaciones educativas en educación infantil. Revista Digital Enfoques Educativos, nº 75, p. 85-94, Mar. 2011.

SANTOS, C. A. DOS; BACINELLO, E.; HEIN, N. Fatores discriminantes dos estilos de aprendizagem dos acadêmicos de ciências contábeis. Revista de Educação, Ciência e Cultura, Canoas, v. 22, n. 3, 2017.

SILVA, G. DO O. L.; WECHSLER, S. M. Estilos de aprendizagem: análise de produção científica brasileira. Revista Estilos de Aprendizaje, v. 5, n. 3, Abril, 2010.

ÜNSAL, G. A Study on the Importance of Learning Styles in Foreign Language Teaching. Journal of Languages' Education and Teaching, v. 6, n. 2, p. 184-191, 2018.

VILLAVICENCIO, L. M. El aprendizaje autónomo en la educación a distancia. 2004. Primer Congreso Virtual Latinoamericano de Educación a Distancia. Disponível em:

LatinEduca2004.com, acesso em dezembro/2019. 